Revista lus et Praxis, Año 18, N² 2, 2012, pp. 333 - 340

ISSN 0717 - 2877

Universidad de Talca - Facultad de Ciencias Jurídicas y Sociales

"Comentario crítico a la investigación de Sebastián Salinero Echeverría

¿¿Por qué aumenta la población penal en Chile?

Un estudio criminológico longitudinal'"

Jean Pierre Matus Acuña

Ma. Carolina Peña y Lillo Tolosa

\title{
COMENTARIO CRÍTICO A LA INVESTIGACIÓN DE SEBASTIÁN SALINERO ECHEVERRÍA “¿POR QUÉ AUMENTA LA POBLACIÓN PENAL EN CHILE? UN ESTUDIO CRIMINOLÓGICO LONGITUDINAL"**
}

\author{
Jean Pierre Matus Acuña** \\ Ma Carolina PeÑa y Lillo Tolosa $^{* * *}$
}

En la Revista lus et Praxis No 1 del año 2012, don Sebastián Salinero Echeverría publicó un artículo denominado "¿Por qué aumenta la población penal en Chile? Un estudio criminológico longitudinal" en donde analiza los factores y las respectivas causas del aumento de la población penal en Chile en la última década.

Lo primero que hay que decir al respecto es que se trata de un esfuerzo notable por el planteamiento del problema, que apunta más allá de la simple constatación estadística del hacinamiento carcelario y las que se consideran como altas tasas de personas privadas de libertad, con lo cual es posible comenzar a discutir seriamente cómo racionalizar el uso de la cárcel, antes que sólo adoptar medidas de carácter paliativo, como las que ha promovido el actual Gobierno, mediante indultos generales, conmutación de penas y una reforma integral de la Ley $N^{\circ} 18.216$.

Según Salinero, el primer factor que incidiría en el aumento de la población penal en Chile corresponde a la disociación entre los flujos de entrada y salida de los recintos penitenciarios, el cual tendría como causas el impacto de la Reforma Procesal Penal y ciertas leyes que favorecen la prisionalización (la multa impaga y delitos de VIF). El segundo factor se relacionaría con los prolongados tiempos de privación de libertad que permanecerían los internos condenados, fenómeno que sería resultado de algunas leyes que agravarían la punición de los delitos contra la propiedad y los relativos al tráfico ilícito de estupefacientes.

\footnotetext{
* Colaboración recibida el 24 de septiembre y aprobada el 22 de octubre de 2012.

** Mg. y Dr. en Derecho por la Universidad Autónoma de Madrid. Becario Post-doctoral Alexander v. Humboldt. Profesor Titular de Derecho penal de la Universidad de Chile. Correo electrónico: jpmatus@ derecho.uchile.cl.

*** Alumna investigadora de la Universidad de Chile.
} 
Y por último, restaría el factor relativo a la falta de mecanismos eficientes que ayuden a descomprimir el sistema carcelario encuentra sus causas en el uso restringido de la libertad condicional y beneficios intrapenitenciarios.

Por otra parte, el autor antes de referirse a los factores y causas del aumento de la población carcelaria, descarta como posible factor el aumento en las tasas de criminalidad al sostener, en base a la tesis del finés Lappi-Seppala, que las tasas de encarcelamiento no guardan relación alguna con los niveles y tendencias de la delincuencia.

Empecemos por esto último. Desde luego, no se trata de una afirmación que pueda compartirse de manera absoluta, al menos no desde que existen importantes e inumerables estudios empíricos que demuestran la dependencia de las tasas de encarcelamiento respecto de la actividad criminal, pues cualquiera sea la forma de funcionamiento de un sistema de justicia criminal, éste responderá a la actividad criminal existente. Por otra parte, la criminología positiva basada en estudios econométricos ha demostrado que las tasas de criminalidad y encarcelamiento guardan estrecha relación como variables dependientes del comportamiento criminal ${ }^{1}$.

En este sentido, una serie de estudios económicos demuestran empirícamente una relación inversamente proporcional entre las variables debatidas, estableciendo que mientras menor sea el tiempo promedio de duración de las condenas efectivas, mayores serán las tasas de criminalidad existentes. Y como el encarcelamiento provoca la incapacitación y con ello, la prevención en la comisión de delitos futuros al ubicar al individuo en prisión, se sitúa a la prisionalización como un factor determinante en las tasas de criminalidad ${ }^{2}$.

\footnotetext{
${ }^{1}$ Véase, en general, para el caso chileno, Matus, Jean Pierre, "¿Por qué no bajan las tasas de criminalidad en Chile?", en el mismo, Derecho penal, criminología y política criminal en el cambio de siglo, Santiago, Ed. Jurídica de Chile, 2011, pp. 177-211.

${ }^{2}$ Existen diversos estudios que determinan una relación causal entre población penal y tasas de delincuencia: BeCKER Gary, "Crime and Punishment: An Economic Approach", Journal of Political Economy, Vol. 76, № 2 (1968); EhrцICH I., "Participation in Illegitimate Activities: A Theoretical and Empirical Investigation", Journal of Political Economy, Vol. 81, № 3 (1973); WITTE, Ann, "Estimating The Economic Model of Crime with Individual Data", Quartely Journal of Economics, Vol. 94 (1980); LevITT, Steve, "The Effect of Prison Population Size on Crime Rates: Evidence from Prison Overcrowding Litigation" The Quarterly Journal of EConomics, Vol. 111, № 2 (1996); KESSLER, Marina; MoLINARI, Andrea, "Una aproximación microeconómica al crimen en la Argentina" Anales de la XXXI Reunión Anual de la Asociación Argentina de Economía Política (1997); AHMED, N.U.; RAHIM, M.A., "A dinamyc model for crime control and policy evaluation", Mathematical problems of Engineering, V. 4 (1998); BANDRés E. y Díez-Tıcıo A., "Delincuencia y acción policial: Un enfoque económico", Revista de Economía Aplicada, Vol. IX, No 27 (2001); LeVITt, Steven D., "Entendiendo por qué se Redujo la Criminalidad en la Década de 1990: Cuatro Factores que lo Explican y Seis que No". Ius et Praxis, Vol. 10, n. 2 (2004); Donohue, John, "Economic Models of Crime and Punishment" en Social Research, 74 (2007); Rivera, Jorge; NúÑEZ, Javier; VILLAVICENCIO, Xávier, "Crimen y Disuasión en Chile, Evidencia desde un modelo de ecuaciones simultáneas para las regiones de Chile" Estudios de Economía 30, Santiago (2002);
} 
Por lo tanto, no basta con negar la validez de los datos de las tasas de denuncia (que, por otra parte, se utilizan en todos los estudios econométricos internacionales) y aferrarse a la ENUSC (que, en todo caso, el año 2011 apuntó un aumento en un 10,3\% respecto del año 2010) para sugerir que en Chile habría bajado la actividad criminal a pesar del aumento en las tasas de encarcelamiento, pues ello no considera el hecho de que la ENUSC no registra los delitos cometidos contra establecimientos comerciales, los cuales representan una cuota importante, sino la mayor, de los delitos contra la propiedad. Eso explica la diferencia que se aprecia con el indicador Casos Policiales determinado por la Subsecretaría de Prevención del Delito que muestra, en los últimos siete años, que en la Región Metropolitana éstos han aumentado sostenidamente de 3.483,8 cada 100.000 habitantes en el año 2005 a 4.297,9 en el año 2011, coincidiendo con el aumento de la población carcelaria año a año ${ }^{3}$.

Luego, parece necesario un estudio más profundo, de carácter empírico, de las relaciones entre la actividad criminal y la población carcelaria en Chile, antes que afirmar su desvinculación teórica con un argumento de autoridad que, en cuanto tal, puede ser también desmentido por otras autoridades con diferente orientación teórica.

En cuanto a los factores que, según Salinero, sí inciden efectivamente en el aumento de las tasas de encarcelamiento, se señala en primer lugar el mayor flujo de ingresos con falta de correspondencia a los egresos, en donde sitúa como una de las causas que lo originaría el impacto de la Reforma Procesal Penal. En este sentido, si bien existe una reducción en el tiempo de duración de los procesos judiciales y un incremento en el número de sentencias definitivas condenatorias, el Sr. Salinero no distingue críticamente el tipo de procedimiento del cual emanan y tampoco menciona que la probabilidad de obtener una sentencia condenatoria que establezca una pena privativa de libertad efectiva es, en nuestro sistema, significativamente baja.

En efecto, del total de términos registrados por el Ministerio Público el año 2011, respecto de imputados conocidos, sólo el 23,63\% correspondió a una sentencia condenatoria, mientras que las salidas alternativas en casos constitutivos de delitos alcanzaron, en conjunto, algo más de un 43\% (suspensión condicional: 29,31\%; acuerdo reparatorio: 2,79\%; principio de oportunidad: 11,28\%). Del resto de los casos con imputado conocido, según el Ministerio Público, un $18,52 \%$ corresponde al archivo provisional, y un $4,59 \%$ a casos de facultad de no perseverar. Finalmente, un $4,35 \%$ de los casos terminó en que el

De La Fuente, Hanns; Mejías, Claudia; Castro, Pía, "Análisis econométrico de los determinantes de la criminalidad en Chile". Polít. crim. Vol. 6, № 11 (2011), entre otros.

${ }^{3}$ Ver http://www.seguridadpublica.gov.cl/ 
Fiscal solicitó al Juez de Garantía la aprobación de una orden de no investigar, por no ser los hechos constitutivos de delito. Y apenas un 4,44\% terminó en situaciones en que se puede suponer una intervención efectiva de la defensa: sentencia absolutoria $(0,76 \%)$; sobreseimiento definitivo $(2,33 \%)$; y sobreseimiento temporal $(1,35 \%)$.

La misma información estadística disponible indica que, del total de las sentencias condenatorias del año 2011 (249.133), sólo 7.191 fueron dictadas en juicios orales, es decir, menos del $3 \%$. Lo que equivale a decir que prácticamente el $97 \%$ de los condenados lo fue en alguno de los procedimientos negociados, a saber, juicio abreviado, juicio simplificado y procedimiento monitorio para faltas. De la información contenida en el Boletín Estadístico del Ministerio Público del año 2011 se puede concluir que en esos procesos negociados, de los 83.954 condenados a penas privativas de libertad, 50.495 recibieron algún beneficio de la Ley $\mathrm{N}^{\circ} 18.216$, lo que representa el $60 \%$ de todos los condenados a penas privativas de libertad, y al menos 157.378 fueron condenados exclusivamente a penas de multa, esto es, el 65\% del total de las sentencias condenatorias (241.332).

Con esta evidencia estadística, es difícil afirmar que el actual Sistema Procesal Penal esté diseñado o al menos se haya implementado para aumentar el número de personas condenadas a penas privativas de libertad. El aumento de las personas efectivamente privadas de libertad parece provenir de otra causa, como plausiblemente lo es el aumento de la actividad criminal, antes que del funcionamiento de nuestro Sistema Procesal Penal, que opera más bien como una válvula para impedir los aumentos de personas presas antes que como un mecanismo de prisionización: un imputado conocido, llevado o no a ACD, durante el año 2011, tiene una probabilidad de no ir a prisión es de un 0,921. O, dicho al revés, la probabilidad de sufrir una pena privativa de libertad el año 2011 en casos con imputados conocidos apenas alcanzó un poco más del $7 \%$.

Sin embargo, siempre es posible decir que si no fuera por el sistema procesal que existe, tal vez las probabilidades de recibir sentencias condenatorias privativas de libertad serían todavía más bajas. No obstante, esta afirmación hipotética no puede ser confirmada empíricamente.

Tampoco tiene sustento empírico la sugerencia que hace Salinero de que entre nosotros la causa del aumento de las tasas de encarcelamiento se originaría en la supuesta existencia de leyes que favorecerían el encarcelamiento y el aumento en los tiempos de privación de libertad. Así, respecto de los presos por multas impagas (art. 49 del Código Penal, hoy modificado para sustituir el pago por trabajos en beneficio de la comunidad), si bien, ha engrosado la población penitenciaria injustificadamente, constituyendo un $13,26 \%$ del sistema cerrado durante el año 2010, el autor no pone énfasis en el tiempo que efectivamente se permanece en prisión por causa de una multa, el cual promedia 6,19 días 
de privación de libertad, conllevando un movimiento constante de personas ingresando y egresando de prisión ${ }^{4}$. De hecho, este fenómeno no sólo se observa en las multas impagas sino que también en los delitos relacionados a violencia intrafamiliar y DMCS, entre otros.

Así se concluye en una investigación dirigida y realizada por los autores de este comentario, respectivamente, gracias a la colaboración de GeNCHI, se obtuvo información relativa al tiempo promedio de duración de las condenas efectivas en Chile de distintos recintos penitenciarios de la Región Metropolitana en los años 2003 y 2008, que demuestra lo contrario a lo afirmado por Salinero, esto es, que en Chile no ha aumentado el tiempo promedio de permanencia en prisión, a pesar de los cambios legislativos que el autor cirticado apunta.

En efecto, realizando un análisis de los datos recibidos, se evidencia una disminución significativa en los tiempos de permanencia en prisión por la comisión de DMCS 5 . A modo de ejemplo, en el CDP Santiago Sur, durante el año 2003, los internos condenados por el delito de homicidio permanecían en promedio un total de 1.448,7 días, mientras que en el año 2008, dicha cifra disminuyó a 747,2 días; y en el delito de lesiones, dentro del mismo recinto, el tiempo promedio de duración de la condena efectiva en el año 2003 era de 1.088,3 días, y en el año 2008 fue de tan sólo 276,6 días.

Es cierto que, como señala Salinero, el legislador ha procurado estas últimas décadas "aumentar" las penas de ciertos delitos, exacerbando aparentemente la respuesta penal en delitos como los relativos a la violencia intrafamiliar, de carácter sexual, funcionarios, algunos contra la propiedad y los de tráfico ilícito de estupefacientes. Sin embargo, una cosa es lo que se establece en la legislación y otra la práctica procesal real, donde factores como las reglas de determinación de las penas, la apreciación cuasiautomática de circunstancias atenuantes en procedimientos negociados, los beneficios de la Ley $N^{\circ} 18.216$ y, posteriormente, la reducción también cuasiautomática de las penas por buena conducta, llevan a que las penas efectivamente impuestas y cumplidas no siempre sean privativas de libertad y, en caso de serlo, que su duración efectiva sea inferior o muy inferior a las previsiones del legislador.

Es por ello que no es posible deducir de la sola lectura de la legislación penal que ésta produzca un aumento tanto del número de encarcelados como del tiempo en que éstos permanecerán en prisión, como sugiere Salinero.

\footnotetext{
${ }^{4}$ WelsCH, Gherman; MuÑoz, Nicolás, "Conceptos № 25: La pena de multa en Chile y su efecto en la población penal", p. 9. Año 2012.

${ }^{5}$ Peña y Liılo, María Carolina, "Tiempo promedio de duración de las condenas en Chile. Análisis de Casos" (en elaboración), Memoria para optar al grado de Licenciado en Ciencias Jurídicas y Sociales, Universidad de Chile, Año 2012. Dir.: JP Matus.
} 
Así, por ejemplo, a diferencia de los planteamientos legales y doctrinarios del autor criticado, la realidad carcelaria de la última década manifiesta una disminución significativa en el tiempo de duración de las condenas en delitos contra la propiedad y diversas infracciones a la Ley de Drogas.

En efecto, siguiendo con el caso paradigmático del CDP Santiago Sur, en este recinto se producen reducciones del tiempo promedio de duración de las condenas significativas, en especial tratándose de delitos contra la propiedad de menor gravedad como el hurto, robo con fuerza y el robo por sorpresa. En el caso del hurto, en este recinto se presenta una disminución de un $87,8 \%$ aproximadamente, en donde al año 2003 los reclusos permanecían 1.318,4 días en promedio por cometer este delito y cinco años después, los ingresos en calidad de condenado presentaban una pena efectiva de sólo 160,4 días, lo que no llega ni a los 6 meses de presidio o reclusión por la comisión de este delito. Otros fenómenos similares dentro del CDP Santiago Sur se producen en los delitos de robo con fuerza y robo por sorpresa que experimentaron reducciones de alrededor $79,3 \%$ y $62,8 \%$, respectivamente. En cuanto al robo con violencia e intimidación, en el año 2003, el tiempo promedio de condena efectiva era de 1.494,1 días, mientras que en el año 2008 disminuyó a 887,5 días. Esta tendencia se presenta en todos los recintos penitenciarios estudiados y respecto de todos los DMCS y delitos relativos al tráfico de drogas y estupefacientes. En relación a estos últimos, en el recinto CDP Santiago Sur, durante el año 2003 los condenados estuvieron cumpliendo una pena efectiva que promediaba los $1.427,3$ días, mientras que en el año 2008 sólo permanecieron recluidos 206,8,días.

Naturalmente, el hecho de que una parte importante de los presos en Chile cumplan condenas por esta clase de delitos no significa que ellas sean las más extensas, sino que es más bien, reflejo de la actividad criminal, tal como se desarrolla en Chile, donde la mayor proporción de delitos cometidos son, precisamente, los relativos a la propiedad y al tráfico de drogas. La extensión de las penas por un delito es, como se ha demostrado, un asunto diferente a la proporción de condenados por ese delito que pueda observarse en un momento determinado.

Por último, el tercer factor aludido por Salinero es la falta de instrumentos adecuados para la descompresión del sistema carcelario.

Al respecto, hay que distinguir: en primer lugar, ya hemos señalado que tal afirmación no es compatible con el uso extensivo de las penas exclusivamente pecuniarias y la vasta aplicación de la Ley $\mathrm{N}^{\circ} 18.216$, en cuya virtud e independiente de las fluctuaciones demográficas consideradas por el autor, la probabilidad de que un condenado originalmente a una pena privativa de libertad la vea sustituida por un beneficio alternativo ha permanecido de manera prácticamente invariable en la franja del $50 \%$ en los últimos diez años. 
Sin embargo, y pese a las consideraciones anteriores y al efecto de la Ley № 18.856, que permite la reducción de penas por buen comportamiento, lleva razón Salinero en destacar la alarmante situación relativa a la baja aplicación de la Libertad Condicional y los beneficios intrapenitenciarios, pues ello parece demostrar que los encargados de su aplicación han hecho suyas las críticas que en la década de 1970 se hacían a los mecanismos de rehabilitación, renunciando a ellos o, al menos, no tomando en cuenta los esfuerzos (en todo caso, todavía insuficientes) que en ese sentido se siguen realizando por Gendarmería de Chile.

En defintiva, podemos decir, contestando a la pregunta que se hace Salinero, que parece más plausible, desde el punto de vista empírico, afirmar que las tasas de encarcelamiento en Chile han aumentado por el aumento de la actividad criminal en los delitos de mayor preferencia, aumento debido, entre otras razones, a que los costos en la decisión de delinquir en esa clase de delitos son muy bajos, dada la baja probabilidad de ser condenado a una pena efectiva de privación de libertad, potenciada por la alta probabilidad de que, en tal caso, la pena que se reciba sea de corta o muy poca duración.

La Reforma Procesal Penal sería, por tanto, en cierta medida responsable de este aumento, pero no por las razones que da Salinero, sino por la contraria: porque asegura en cortos tiempos de tramitación procesal que los imputados no recibirán pena alguna o, en caso de recibirla, ésta será de corta duración.

A pesar de las diferencias apuntadas, agradecemos al Sr. Salinero la oportunidad de plantear este debate que, estimamos, permitirá una mejor comprensión del funcionamiento real de nuestro sistema penal. 
